\title{
Two new species of Hepatozoon (Apicomplexa: Hepatozoidae) parasitising species of Philothamnus (Ophidia: Colubridae) from South Africa
}

\author{
Courtney Antonia Cook ${ }^{1,2}$, Edward Charles Netherlands ${ }^{2,3}$, Johann van As ${ }^{1}$ and Nico Jacobus Smit ${ }^{2}$ \\ ${ }^{1}$ Department of Zoology and Entomology, University of the Free State, Qwaqwa Campus, Phuthaditjhaba, South Africa; \\ ${ }^{2}$ Water Research Group, Unit for Environmental Sciences and Management, North-West University, Potchefstroom, South Africa; \\ ${ }^{3}$ Laboratory of Aquatic Ecology, Evolution and Conservation, University of Leuven, Leuven, Belgium
}

\begin{abstract}
To date, only a few species of Hepatozoon Miller, 1908 have been described from amphibians and reptiles of South Africa, including two species from anuran hosts, three from saurians, one from chelonians, and two from ophidians. Hepatozoon bitis (Fantham, 1925) and Hepatozoon refringens (Sambon et Seligmann, 1907), parasitising Bitis arientans (Merrem) and Pseudoaspis cana (Linnaeus), respectively, were described in the early 1900s and since then there have been no further species of Hepatozoon described from snakes in South Africa. Blood smears, used in peripheral blood haemogregarine stage morphometrics, and whole blood used in molecular characterisation of haemogregarines were collected from the caudal vein of six snakes of three species, namely Philothamnus hoplogaster (Günther), Philothamnus semivariegatus (Smith) and Philothamnus natalensis natalensis (Smith). For comparison, a comprehensive table summarising available information on species of Hepatozoon from African snakes is presented. Haemogregarines found infecting the snakes from the present study were morphologically and molecularly different from any previously described from Africa and are thus here described as Hepatozoon angeladaviesae sp. n. and Hepatozoon cecilhoarei sp. n. Both haemogregarine species were observed to cause considerable dehaemoglobinisation of the host cell, in case of infection with $H$. angeladaviesae resulting in a characteristic peripheral undulation of the host cell membrane and karyorrhexis. To the authors' knowledge, these are the first haemogregarines parasitising snakes of the genus Philothamnus Smith described using both morphological and molecular characteristics in Africa.
\end{abstract}

Keywords: serpents, snakes, haemogregarines, phylogeny, adeleorid taxonomy, 18S rDNA, haemoparasites

Haemogregarines of the genus Hepatozoon Miller, 1908 (Hepatozoidae) are intraerythrocytic or intraleucocytic apicomplexan parasites that are frequently described from amphibian and reptilian hosts (Smith 1996, Cook et al. 2014a). However, as highlighted by Borges-Nojosa et al. (2017), the diversity and systematics of these apicomplexans are still poorly understood. The genus Hepatozoon is paraphyletic based on estimated relationships using $18 \mathrm{~S}$ rRNA gene sequences, the genus Karyolysus Labbé, 1894, and in some analyses Hemolivia Petit, Landau, Baccam et Lainson, 1990, as well, forming a lineage within Hepatozoon (see Barta et al. 2012, Haklová-Kočíková et al. 2014, Kvičerová et al. 2014, Cook et al. 2016).

Recently, a new genus, Bartazoon Karadjian, Chavatte et Landau, 2015, was erected as part of a taxonomic revision to try resolve the phylogeny and associated taxonomy of these haemogregarines, the new genus including species from reptiles that were previously included in Hepatozoon (see Karadjian et al. 2015). However, the monophyly of
Bartazoon is not well supported and with all the molecular evidence provided for by the use of a single gene it is at this time premature to consider such taxonomic changes (Maia et al. 2016, Borges-Nojosa et al. 2017). Thus, following Borges-Nojosa et al.'s (2017) recommendation we will conservatively persist in referring to species parasitising reptiles as Hepatozoon.

To date, only a handful of species of Hepatozoon have been described from amphibians and reptiles of South Africa. From amphibians these include Hepatozoon theileri (Laveran, 1905) infecting the common river frog Amietia delalandii (Bocage) (Pyxicephalidae), Hepatozoon ixoxo Netherlands, Cook et Smit, 2014 infecting typical toads Sclerophrys pusilla (Hallowell), Sclerophrys garmani (Meek) and Sclerophrys gutturalis (Power) (Bufonidae), and recently Hepatozoon involucrum Netherlands, Cook et Smit, 2017 infecting Hyperolius marmoratus Rapp, Hepatozoon tenuis Netherlands, Cook et Smit, 2017 infecting Afrixalus fornasinii (Bianconi), Hyperolius argus Peters

Address for correspondence: C.A. Cook, Water Research Group, Unit for Environmental Sciences and Management, North-West University, Potchefstroom Campus, Potchefstroom, 2531, South Africa. Phone: +27 182992493; E-mail: apicomplexan@yahoo.co.za

Zoobank number for article: urn:lsid:zoobank.org:pub:4D0DB4B5-213A-46A0-93EF-C299E266104F 
and Hyp. marmoratus and Hepatozoon thori Netherlands, Cook et Smit, 2017 infecting Hyp. marmoratus, Hyp. argus and Hyperolius puncticulatus (Pfeffer). From reptiles these include Hepatozoon langii Van As, Davies et Smit, 2013, and Hepatozoon vacuolatus Van As, Davies et Smit, 2013, from crag lizards Pseudocordylus langi Loveridge (Cordylidae), Hepatozoon affluomaloti Van As, Davies et Smit, 2015 from crag lizards Pseudocordylus melanotus Smith, and Pseudocordylus subviridis (Smith) (Cordylidae), Hepatozoon varani (Laveran, 1905) from monitor lizards Varanus niloticus (Linnaeus) (Varanidae), and Hepatozoon fitzsimonsi (Dias, 1953) from five species of terrestrial chelonians including Chersina angulata (Schweigger), Kinixys lobatsiana Power, Kinixys natalensis Hewitt, Kinixys zombensis Hewitt and Stigmochelys pardalis (Bell) (Testudinidae) (see Laveran 1905, Dias 1953, Smith 1996, Cook et al. 2009, 2014a, 2016, Van As et al. 2013, 2015, Netherlands et al. 2014a,b, 2018).

Even though numerous species of Hepatozoon have been described from snakes throughout Africa from various families including Colubridae, Elapidae, Lamprophiidae, Natricidae, Pythonidae and Viperidae (Table 1), the only species of Hepatozoon described to date from South African snakes are Hepatozoon bitis (Fantham, 1925) described from Bitis arietans (Merrem) (Viperidae) and Hepatozoon refringens (Sambon and Seligmann, 1907) from Pseudoaspis cana (Linnaeus) (Lamprophiidae) (Sambon and Seligmann 1907, Fantham 1925).

Within the Colubridae, snakes of the genus Philothamnus Smith, frequently formed part of haemoparasite surveys in Africa, these reporting infections of potentially several species of haemogregarines. However, regardless of the sometimes detailed descriptions of these Hepatozoon spp., none were ever named (Bouet 1909, Hoare 1920, Schweitz 1931, Garnham and Duke 1953, Ball 1967, Haklová et al. 2014).

As part of a larger project focusing on haemoparasites of South African reptiles and amphibians, two different types of Hepatozoon were found in the peripheral blood of three species of Philothamnus. Thus, this paper presents the first formal description of species of Hepatozoon parasitising species of Philothamnus in South Africa based on both morphological description and molecular characterisation.

\section{MATERIALS AND METHODS}

\section{Snake collection and blood preparation}

A total of six snakes were collected, including two Philothamnus hoplogaster (Günther), two Philothamnus natalensis natalensis (Smith) and two Philothamnus semivariegatus (Smith), in the Ndumo Game Reserve, KwaZulu-Natal (32¹8'49"E; 2654'33"S) (see fig. 1 in Netherlands et al. 2015) from 2014-2016 (Permit no. OP $839 / 2014)$. Blood from the caudal vein was aspirated into a sterile $1 \mathrm{ml}$ insulin syringe. Thin blood smears were prepared, air-dried, fixed for $10 \mathrm{~min}$ in absolute methanol and stained using a modified solution of Giemsa stain (Sigma-Aldrich, Steinheim, Germany) for 20 min following Cook et al. (2014a, 2016). A small volume $(<0.5 \mathrm{ml})$ of blood from each specimen was also dropped into molecular grade 70\% ethanol (Sigma-Aldrich) for molecular analyses.

\section{Blood screening}

Smears were screened using a $100 \times$ oil immersion objective, and micrographs and measurements of parasites were taken on a calibrated Nikon Eclipse E800 compound microscope (Nikon, Amsterdam, Netherlands) using the Nikon NIS-Elements microscope imaging software program D3.2 (Nikon). All measurements are in micrometres $(\mu \mathrm{m})$ unless otherwise indicated. Parasitaemia was calculated per 100 erythrocytes, with $\sim 10^{4}$ erythrocytes examined per blood smear (Cook et al. 2014a, 2016).

DNA extraction, PCR and phylogenetic analysis of $18 \mathrm{~S}$ rDNA

Whole blood from one P. hoplogaster, one P. semivariegatus and one $P$. $n$. natalensis, representing microscopically-identified infections of a single morphotype, along with whole blood from one $P$. $n$. natalensis representing a microscopically-identified infection of both morphotypes, were used for DNA extraction following the standard protocol method for human or animal tissue and cultured cells as detailed in the NucleoSpin ${ }^{\circledR}$ Tissue Genomic DNA Tissue Kit (Macherey-Nagel, Duren, Germany).

Molecular characterisation of parasites was performed via PCR amplification, amplifying approximately the full $18 \mathrm{~S}$ rRNA gene in two fragments by using a combination of primer sets. The first fragment, approximately $930 \mathrm{nt}$ in length, was amplified using primer set HAMF 5'-GCCAGTAGTCATATGCTTGTC-3' (Criado-Fornelio et al. 2006) and HepR900 5'-CAAATCTAAGAATTTCACCTCTGAC-3' (Ujvari et al. 2004). The second fragment, approximately $1,400 \mathrm{nt}$ in length, was amplified using primer set HepF300 5'-GTTTCTGACCTATCAGCTTTCGACG-3' (Ujvari et al. 2004) and 2868 5'-TGATCCTTCTGCAGGTTCAC-3' (Medlin et al. 1988, Mathew et al. 2000).

Conditions for PCR of both fragments were as follows: initial denaturation at $95^{\circ} \mathrm{C}$ for $3 \mathrm{~min}$, followed by 35 cycles, entailing a $95^{\circ} \mathrm{C}$ denaturation for $30 \mathrm{~s}$, annealing at $61^{\circ} \mathrm{C}$ for $30 \mathrm{~s}$ with an end extension at $72^{\circ} \mathrm{C}$ for $2 \mathrm{~min}$, and following the cycles a final extension of $72^{\circ} \mathrm{C}$ for $10 \mathrm{~min}$ (Netherlands et al. 2018).

All PCR reactions were performed with volumes of $25 \mu 1$, using $12.5 \mu 1$ Thermo Scientific DreamTaq PCR master mix $(2 \times)$ ( $2 \times$ DreamTaq buffer, $0.4 \mathrm{mM}$ of each dNTP, and $4 \mathrm{mM} \mathrm{MgCl}_{2}$ ), $1.25 \mu \mathrm{l}$ of each primer $(10 \mu \mathrm{M})$, and at least $25 \mathrm{ng}$ of DNA. PCR grade nuclease free water (Thermo Scientific, Vilnius, Lithuania) was used to make up final reaction volume. Reactions were undertaken in a Bio-Rad C1000 Touch ${ }^{\mathrm{TM}}$ Thermal Cycler PCR machine (Bio-Rad, Hemel Hempstead, UK). An agarose gel (1\%) stained with gel red was used to visualise resulting amplicons under UV light.

Two PCR products from each sample were sent to a commercial sequencing company (Inqaba Biotechnical Industries (Pty) Ltd., Pretoria, South Africa) for purification and sequencing in both directions. Quality of resultant sequences was assessed using Geneious Ver. 7.1 (http://www.geneious.com, Kearse et al. 2012) before consensus sequences were generated from both forward and reverse sequence reads for both fragments. A consensus sequence was then generated from both fragments, with an overlap of $\sim 600 \mathrm{nt}$ (with 100\% identity). Sequences were identified 
Table 1. Haemogregarines of the genus Hepatozoon Miller, 1908 (Apicomplexa: Hepatozoidae) described from snakes from Africa. Parasite species and authorities, snake host (family), localities (type and other), mature gamont and gamont nucleus description (measurements in $\mu \mathrm{m})$, and references are provided.

\begin{tabular}{|c|c|c|c|c|}
\hline Parasite species and authority & Snake host (family) & Locality & $\begin{array}{l}\text { Description } \\
\text { (gamont; nucleus) }\end{array}$ & References \\
\hline $\begin{array}{l}\text { Hepatozoon aegypti Bashtar, Boulos, } \\
\text { et Mehlhorn, } 1984\end{array}$ & $\begin{array}{l}\text { Spalerosophis diadema (Schlegel) (Col- } \\
\text { ubrida) }\end{array}$ & Type: Egypt & $19-21.5 \times 1.7-2.8 ;-$ & $\begin{array}{l}\text { Bashtar et al. 1984, Morsy } \\
\text { et al. } 2013\end{array}$ \\
\hline Hepatozoon algiri (Manceaux, 1908) & Platyceps sp. (Colubridae) & Type: Algeria & $19 \times 4 ;-$ & Manceaux 1908 \\
\hline Hepatozoon angeladaviesae $\mathrm{sp} . \mathrm{n}$. & $\begin{array}{l}\text { Philothamnus semivariegatus (Smith), } \\
\text { Philothamnus hoplogaster (Günther), } \\
\text { Philothamnus natalensis natalensis } \\
\text { (Smith) (Colubridae) }\end{array}$ & $\begin{array}{l}\text { Type: South } \\
\text { Africa }\end{array}$ & $\begin{array}{l}14.1-17.3 \times 4.8-6.5 \\
3.6-5.7 \times 3.7-5.8\end{array}$ & Present study \\
\hline $\begin{array}{l}\text { Haemogregarina arabi Ramadan, } 1974 \\
\text { (likely Hepatozoon arabi) }\end{array}$ & Telescopus dhara (Forskal) (Colubridae) & Type: Egypt & $11-18 \times 3-5.5$ & Mohammad et al. 1996 \\
\hline $\begin{array}{l}\text { Haemogregarina aswanensis Moham- } \\
\text { mad, Ramdan, Mohammed et Fawzi, } \\
1996 \text { (likely Hepatozoon aswanensis) }\end{array}$ & Naja haje (Linnaeus) (Elapidae) & Type: Egypt & $\begin{array}{l}12.5-17.5 \times 2.5-6 \\
3.7 \times 2.6\end{array}$ & Mohammad et al. 1996 \\
\hline $\begin{array}{l}\text { Hepatozoon ayorgbor Sloboda, Kamler, } \\
\text { Bulantová, Votýpka et Modrý, } 2007\end{array}$ & ; Python regius (Shaw) (Py & Type: Ghana & $\begin{array}{l}11-13 \times 2-3.5 \\
4-6.5 \times 1.5-2\end{array}$ & Sloboda et al. 2007 \\
\hline Hepatozoon bitis (Fantham, 1925) & Bitis arietans Merrem (Viperidae) & $\begin{array}{l}\text { Type: South } \\
\text { Africa }\end{array}$ & $12.5-14 \times 3-4 ;-$ & $\begin{array}{l}\text { Fantham } 1925 \text {, Hoare } \\
\text { 1932, Smith 1996, Slobo- } \\
\text { da et al. } 2007\end{array}$ \\
\hline Hepatozoon boodoni (Phisalix, 1914) & $\begin{array}{l}\text { Boaedon fuliginosus (Boie) (Lamprophii- } \\
\text { dae) }\end{array}$ & Type: Sudan & $\begin{array}{l}14-15 \times 2-3, \\
14-15 \times 7 ; 5\end{array}$ & $\begin{array}{l}\text { Phisalix 1914, Smith } \\
\text { 1996, Sloboda et al. } 2007\end{array}$ \\
\hline $\begin{array}{l}\text { Hepatozoon brendae (Sambon et } \\
\text { Seligmann, 1907) }\end{array}$ & $\begin{array}{l}\text { Psammophis sibilans (Linnaeus) (Lam- } \\
\text { prophiidae) }\end{array}$ & $\begin{array}{l}\text { Type: Tropical } \\
\text { Africa and Egypt }\end{array}$ & $\begin{array}{l}16-17 \times 3-4 \\
5-6 \times 3-4\end{array}$ & $\begin{array}{l}\text { Sambon and Seligmann } \\
1907 \text {, Smith 1996, Slobo- } \\
\text { da et al. } 2007\end{array}$ \\
\hline Hepatozoon cecilhoarei sp. n. & $\begin{array}{l}\text { Philothamnus natalensis natalensis }{ }^{1} \\
\text { Philothamnus hoplogaster }{ }^{1} \text {, Philotham- } \\
\text { nus } \text { sp. }^{2} \text { (Colubridae) }\end{array}$ & $\begin{array}{l}\text { Type: South } \\
\text { Africa } \\
\text { Other: Uganda }{ }^{2}\end{array}$ & $\begin{array}{l}13.1-15.9 \times 2.1-2.7 \\
4.2-5.8 \times 1.2-1.6\end{array}$ & $\begin{array}{l}\text { Present study }{ }^{1}, \text { Hoare } \\
1920^{2}\end{array}$ \\
\hline $\begin{array}{l}\text { Hepatozoon crotaphopeltis (Hoare, } \\
\text { 1932) }\end{array}$ & $\begin{array}{l}\text { Crotaphopeltis hotamboeia (Laurenti) } \\
\text { (Colubridae) }\end{array}$ & Type: Uganda & $20 \times 2 ;-$ & $\begin{array}{l}\text { Hoare 1932, Smith 1996, } \\
\text { Sloboda et al. } 2007\end{array}$ \\
\hline Hepatozoon dogieli (Hoare, 1920) & $\begin{array}{l}\text { Bitis gabonica Duméril, Bibron et } \\
\text { Duméril (Viperidae) }\end{array}$ & Type: Uganda & $14 \times 6 ;-$ & $\begin{array}{l}\text { Hoare 1932, Smith 1996, } \\
\text { Sloboda et al. } 2007\end{array}$ \\
\hline Hepatozoon enswerae (Hoare, 1932) & Naja melanoleuca Hallowell (Elapidae) & Type: Uganda & $\begin{array}{l}19 \times 3, \text { slender } \\
\text { vermicular } 15 \times 3.8 \\
\text { bean-shaped; - }\end{array}$ & $\begin{array}{l}\text { Hoare 1932, Smith 1996, } \\
\text { Sloboda et al. } 2007\end{array}$ \\
\hline $\begin{array}{l}\text { Hepatozoon garnhami (Mohammad, } \\
\text { Ramdan, Mohammed et Fawzi, 1996) }\end{array}$ & $\begin{array}{l}\text { Psammophis aegyptius Marx (Lam- } \\
\text { prophiidae) }\end{array}$ & Type: Egypt & $\begin{array}{l}15-20 \times 1.5-2.5 \\
5-9 \times 1.5-3\end{array}$ & $\begin{array}{l}\text { Mohammad et al. 1996, } \\
\text { Abdel-Baki et al. } 2014\end{array}$ \\
\hline $\begin{array}{l}\text { Hepatozoon joannoni (Hagenmuller, } \\
1898 \text { ) }\end{array}$ & $\begin{array}{l}\text { Macroprotodon cucullatus (Geof- } \\
\text { froy-St-Hilaire) (Colubridae) }\end{array}$ & $\begin{array}{l}\text { Type: South } \\
\text { Europe or North } \\
\text { Africa }\end{array}$ & 12-18 in length; - & $\begin{array}{l}\text { Sambon and Seligmann } \\
\text { 1907, Smith } 1996\end{array}$ \\
\hline $\begin{array}{l}\text { Hepatozoon malpoloni (Ramadan, } \\
\text { 1974) }\end{array}$ & $\begin{array}{l}\text { Malpolon monspessulanus (Hermann) } \\
\text { (Lamprophiidae) }\end{array}$ & Type: Egypt & $12-25 \times 3-5.5 ;-$ & $\begin{array}{l}\text { Mohammad et al. 1996, } \\
\text { Smith } 1996\end{array}$ \\
\hline $\begin{array}{l}\text { Hepatozoon matruhensis Shazly, } \\
\text { Ahmed, Bashtar et Fayed, } 1994\end{array}$ & Psammophis schokari (Lamprophiidae) & Type: Egypt & $18-28 \times 2.5-6 ;-$ & $\begin{array}{l}\text { Mohammad et al. } 1996 \text {, } \\
\text { Smith } 1996\end{array}$ \\
\hline $\begin{array}{l}\text { Hepatozoon mehlhorni Bashtar, Ab- } \\
\text { del-Ghaffar et Shazly, } 1991\end{array}$ & Echis carinatus (Schneider) (Viperidae) & Type: Egypt & $\begin{array}{l}17.2 \times 5.4 \\
6.3 \times 5.4\end{array}$ & $\begin{array}{l}\text { Bashtar et al. 1991, Smith } \\
\text { 1996, Morsy et al. } 2013\end{array}$ \\
\hline Hepatozoon minchini (Garnham, 1950) & $\begin{array}{l}\text { Crotaphopeltis degeni (Boulenger) } \\
\text { (Colubridae) }\end{array}$ & Type: Kenya & $13-14 \times 3-4 ;-$ & $\begin{array}{l}\text { Garnham 1950, Smith } \\
\text { 1996, Sloboda et al. } 2007\end{array}$ \\
\hline Hepatozoon musotae (Hoare, 1932) & $\begin{array}{l}\text { Boaedon sp. Duméril, Bibron et Duméril } \\
\text { (Lamprophiidae) }\end{array}$ & Type: Uganda & $\begin{array}{l}17 \times 3.8-4.7 \text {, bean- } \\
\text { shaped } 15.2 \times 6.6 \\
\text { broad forms; - }\end{array}$ & $\begin{array}{l}\text { Hoare 1932, Smith 1996, } \\
\text { Sloboda et al. } 2007\end{array}$ \\
\hline Hepatozoon najae (Laveran, 1902) & 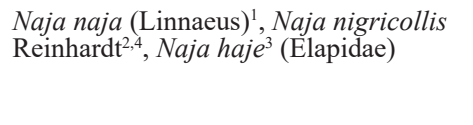 & $\begin{array}{l}\text { Type: } \text { India }^{1} \\
\text { Other: Egypt } \\
\text { Kenya } \\
\text { nia }^{4} \text {, Tanza- }\end{array}$ & $\begin{array}{l}14 \times 3 \text { (folded) } \\
21-22 \times 3 \text { (when not } \\
\text { folded } 1) ;-\end{array}$ & $\begin{array}{l}\text { Laveran } 1902^{1} \text {, Ball } 1967 \text {, } \\
\text { Bashtar and Abdel-Ghaf- } \\
\text { far } 1987^{2} \text {, Smith } 1996 \text {, } \\
\text { Telford } 2009^{4}\end{array}$ \\
\hline $\begin{array}{l}\text { Hepatozoon refringens (Sambon et } \\
\text { Seligmann, 1907) }\end{array}$ & $\begin{array}{l}\text { Pseudaspis cana (Linnaeus) (Lam- } \\
\text { prophiidae) }\end{array}$ & $\begin{array}{l}\text { Type: South } \\
\text { Africa }\end{array}$ & $10-12 \times 5-6 ;-$ & $\begin{array}{l}\text { Sambon and Seligmann } \\
\text { 1907, Smith } 1996\end{array}$ \\
\hline $\begin{array}{l}\text { Hepatozoon robertsonae (Sambon, } \\
\text { 1909) }\end{array}$ & $\begin{array}{l}\text { Python regius, Python sebae (Gmelin) } \\
\text { (Pythonidae) }\end{array}$ & Type: Gambia & 12-16 in length; - & $\begin{array}{l}\text { Sambon and Seligmann } \\
\text { 1907, Sloboda et al. } 2007\end{array}$ \\
\hline $\begin{array}{l}\text { Hepatozoon sebai (Laveran et Pettit, } \\
\text { 1909) }\end{array}$ & Python sebae (Pythonidae) & Type: Senegal & $\begin{array}{l}11-13 \times 2 \text { (folded) } \\
17-18 \times 2 \text { (when not } \\
\text { folded }) ;-\end{array}$ & $\begin{array}{l}\text { Laveran and Pettit 1909, } \\
\text { Smith } 1996\end{array}$ \\
\hline $\begin{array}{l}\text { Hepatozoon seurati (Laveran et Pettit, } \\
\text { 1911) }\end{array}$ & Cerastes cerastes Linnaeus (Viperidae) & $\begin{array}{l}\text { Type: Algeria } \\
\text { Other: Egypt }\end{array}$ & $\begin{array}{l}12-16.5 \times 2-3.5 \\
4.5 \times 3.5\end{array}$ & $\begin{array}{l}\text { Laveran and Pettit 1911, } \\
\text { Smith 1996, Morsy et al. } \\
2013\end{array}$ \\
\hline $\begin{array}{l}\text { Haemogregarina vaughani Balfour, } \\
1908 \text { (likely Hepatozoon vaughani) }\end{array}$ & $\begin{array}{l}\text { Rhamphiophis rubropunctatus (Fischer) } \\
\text { (Lamprophiidae) }\end{array}$ & Type: Sudan & $15 \times 4.5 ;-$ & Balfour 1908 \\
\hline Hepatozoon viperini (Billet, 1904) & Natrix maura (Linnaeus) (Natricidae) & Type: Algeria & None provided & $\begin{array}{l}\text { Billet 1904, Smith 1996, } \\
\text { Sambon and Seligmann } \\
1907\end{array}$ \\
\hline Hepatozoon vubirizi (Hoare, 1932) & $\begin{array}{l}\text { Gonionotophis savorgnani (Mocquard) } \\
\text { (Lamprophiidae) }\end{array}$ & Type: Uganda & $15-17 \times 3.8-4.7 ;-$ & $\begin{array}{l}\text { Hoare 1932, Smith 1996, } \\
\text { Sloboda et al. } 2007\end{array}$ \\
\hline
\end{tabular}


Table 1. Continued.

\begin{tabular}{|c|c|c|c|c|}
\hline Parasite species and authority & Snake host (family) & Locality & $\begin{array}{l}\text { Description } \\
\text { (gamont; nucleus) }\end{array}$ & References \\
\hline Hepatozoon zambiensis (Peirce, 1984) & Dispholidus typus (Smith) (Colubridae) & Type: Zambia & $\begin{array}{l}14.9-17.8 \times 2.4-5.7 \\
4.4-8.4 \times 2.2-4.1\end{array}$ & $\begin{array}{l}\text { Peirce 1984, Smith 1996, } \\
\text { Sloboda et al. } 2007\end{array}$ \\
\hline Hepatozoon zamenis (Laveran, 1902) & $\begin{array}{l}\text { Hemorrhois hippocrepis (Linnaeus) } \\
\text { (Colubridae) }\end{array}$ & Type: Algeria & $18 \times 4 ;-$ & $\begin{array}{l}\text { Laveran 1902, Sambon } \\
\text { and Seligmann } 1907, \\
\text { Smith } 1996\end{array}$ \\
\hline Hepatozoon zumpti (Dias, 1952) & $\begin{array}{l}\text { Dendroaspis polylepis Günther } \\
\text { (Elapidae) }\end{array}$ & $\begin{array}{l}\text { Type: Mozam- } \\
\text { bique }\end{array}$ & $\begin{array}{l}14.3-16.3 \times 4.3-5.3 \\
4.8-5.8 \times 3.8-4.5\end{array}$ & Dias 1952, Smith 1996 \\
\hline
\end{tabular}

using the Basic Local Alignment Search Tool (BLAST) (http:// blast.ncbi.nlm.nih.gov/), and deposited in the NCBI GenBank database under the accession numbers: MG519501-MG519504.

For the phylogenetic analysis comparative sequences of species of Hemolivia, Hepatozoon and Karyolysus, with Haemogregarina balli Paterson et Desser, 1976 (GenBank: HQ224959) as outgroup, were downloaded from GenBank and aligned to the sequences generated within this study. Sequences were aligned using the MUSCLE alignment tool (Edgar 2004) implemented in Geneious Ver. 7.1. The alignment consisted of 34 sequences and was $945 \mathrm{nt}$ long. To infer phylogenetic relationships of the aligned dataset both Maximum Likelihood (ML) and Bayesian Inference (BI) methods were used.

A model test was performed to determine the most suitable nucleotide substitution model, according to the Akaike information criterion using jModelTest 2.1.7 (Guindon and Gascuel 2003, Darriba et al. 2012). The best model identified was the General Time Reversible model with estimates of invariable sites and a discrete Gamma distribution $(\mathrm{GTR}+\mathrm{I}+\Gamma)$. The ML analysis was performed using RAxML Ver. 7.2.8 implemented from within Geneious 7.1. The alphaparameter selected was the GTR GAMMA I model, with support assessed using 500 rapid bootstrap inferences. The BI analysis was implemented from within Geneious 7.1 using MrBayes 3.2.2 (Huelsenbeck and Ronquist 2001).

The analysis was run twice over 10 million generations for the Markov Chains Monte Carlo (MCMC) algorithm. The Markov chain was sampled every 100 cycles, and the MCMC variant contained 4 chains with a temperature of 0.2 . The log-likelihood values of the sample point were plotted against the generation time and the first $25 \%$ of the trees were discarded as 'burn-in' with no 'burn-in' samples being retained. Results were visualised in Trace (implemented from within Geneious) to assess convergence and the 'burn-in' period. As the topologies for both the ML and BI trees were identical resulting trees were combined in a $50 \%$ majority consensus tree.

To compare sequences of isolates of Hepatozoon representing fragments of the $\sim 860-1,725 \mathrm{nt}$ region of the $18 \mathrm{~S}$ rRNA gene from other African snakes, an uncorrected pair-wise distances (p-distance) matrix was used. Comparative sequences (KC800702, KC800703, KC866369, KC866368, KC866370, KC800704, JQ746622) were downloaded from GenBank and aligned to the sequences generated within this study. Sequences were aligned using the MUSCLE alignment tool (Edgar 2004) implemented in Geneious Ver. 7.1. and manually trimmed. The alignment consisted of nine sequences and was $736 \mathrm{nt}$ long when imported into the MEGA7 bioinformatics software program (Kumar et al. 2016) in which the matrix was produced.
Ethics statement. This study received the relevant ethical approval (North-West University ethics approval: NWU-0000514-S3, NWU-00372-16-A5).

\section{RESULTS}

\section{General observations for peripheral blood} developmental stages of Hepatozoon species Fig. 1

All six individuals, two each, of Philothamnus hoplogaster, $P$. semivariegatus and $P$. $n$. natalensis from Ndumo Game Reserve, KwaZulu-Natal, were found to be parasitised by two morphologically dissimilar species of haemogregarines (Fig. 1). The first species, Hepatozoon sp. morphotype A, was found parasitising one P. hoplogaster (parasitaemia $0.01 \%$ ), both $P$. semivariegatus (parasitaemia $0.5 \%$ and $5 \%$, respectively) and one $P$. n. natalensis (parasitaemia 3\%). This latter infection in $P$. n. natalensis was a co-infection with the second haemogregarine morphotype, Hepatozoon sp. morphotype B (parasitaemia $0.01 \%$ ). Only Hepatozoon sp. morphotype B was found parasitising the second specimen of $P$. hoplogaster (parasitaemia $0.2 \%$ ) and the second P. n. natalensis (parasitaemia $1 \%$ ). Intraerythrocytic mature gamonts were found to be the only stages observed within the peripheral blood from all snake specimens and caused notable host cell alterations. The infection with Hepatozoon sp. morphotype A resulted in a cytopathology characterised by an enlarged and dehaemoglobinised host cells with a 'wafer-thin' undulating cell membrane, whilst the infection with Hepatozoon sp. morphotype B was characterised by the parasitised host cell's elongation and narrowing.

No potential vectors, such as mosquitoes or ticks, were found feeding on the snakes.

\section{Molecular identification and phylogenetic analysis}

Amplicons of between 1,540 nt and 1,713 nt of the 18S rRNA gene were obtained for both species of Hepatozoon identified by microscopy. Hepatozoon sp. morphotype A was amplified from one $P$. hoplogaster (1,540 nt), one $P$. semivariegatus $(1,540 \mathrm{nt})$ and one $P . n$. natalensis $(1,540 \mathrm{nt})$ (the specimen representing a co-infection with both morphotypes). All three amplicons were identical from which a consensus sequence was made (1,540 nt). Hepatozoon sp. morphotype B was amplified from one $P$. n. natalensis $(1,713 \mathrm{nt})$ (the specimen representing an infection with only morphotype B). Both the phylogenetic analysis (Fig. 2) and the evolutionary divergence estimates (Table 2) differentiate Hepatozoon sp. morphotype A and 


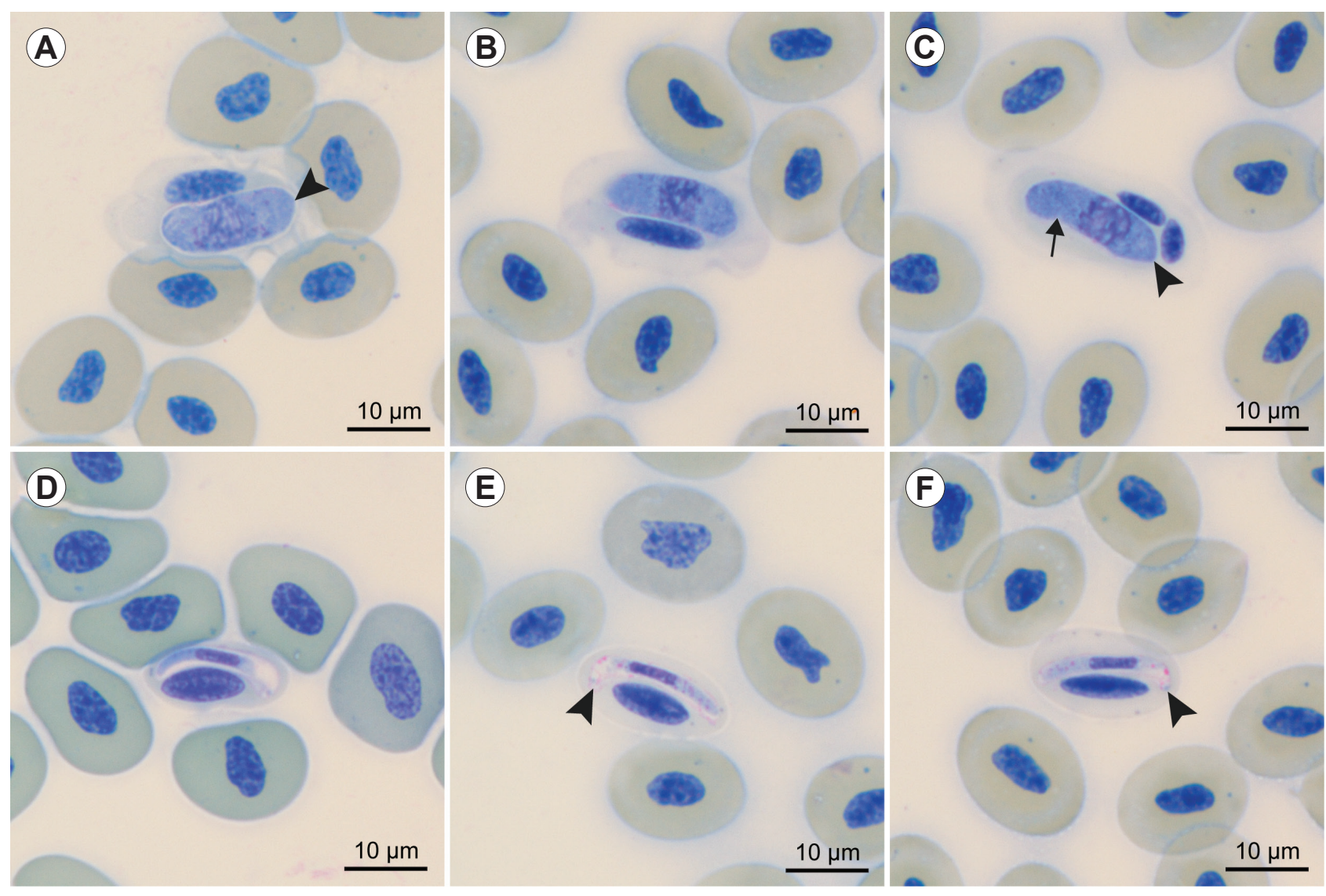

Fig. 1. Peripheral blood stages of two species of Hepatozoon Miller, 1908 parasitising species of Philothamnus Smith. Mature gamonts, lying singly, within a parasitophorous vacuole, within mature erythrocytes of (A-C) Hepatozoon angeladaviesae sp. n. (NMB P 440) parasitising Philothamnus semivariegatus (Smith), and (D-F) Hepatozoon cecilhoarei sp. n. (NMB P 441) parasitising Philothamnus natalensis natalensis (Smith), both from Ndumo Game Reserve, KwaZulu-Natal. A-C - gamont straighter than curved, tapering to a point at one pole (arrowhead), the other pole rounded, sometimes appearing folded (arrow), the parasitophorous vacuole appearing as a halo-like sheath; D-F - gamont elongated and curved, parasitophorous vacuole evident, appearing noticeably larger than the gamont with one pole curved into a hook (arrowhead). In both $H$. angeladaviesae sp. n. (A-C) and H. cecilhoarei sp. n. (D-F), the host cell nucleus has been displaced and condensed, the gamont of $H$. angeladaviesae sp. $n$. causing karyolysis of the host cell nucleus (C). Both haemogregarine species cause dehaemoglobinisation, H. angeladaviesae sp. n. characteristically causing the 'wafer-thin' undulation of the host cell membrane $(\mathrm{A}-\mathrm{C})$.

Hepatozoon sp. morphotype B as separate species, which are described below as Hepatozoon angeladaviesae sp. $\mathrm{n}$. and Hepatozoon cecilhoarei sp. n., respectively. Both species of Hepatozoon are closely related, as can be seen in the phylogenetic analysis and divergence estimates $(99.5 \%$ identical, $\mathrm{p}=0.01$, representing the $\sim 860-1,725 \mathrm{nt}$ region, see Table 2; and $99.0 \%$ identical, $p=0.01$, representing the full 1,540 nt fragment). With high support $(73,0.89)$ both species form a sister clade to Hepatozoon sipedon Smith, Desser et Martin, 1994 from the snake Nerodia sipedon sipedon (Linnaeus), this clade in turn clustering within a larger monophyletic clade, sister to a clade containing species of Hepatozoon from amphibians.

According to the evolutionary divergence estimates concerning species of Hepatozoon from African snakes, which could not be included in the phylogenetic analysis as they represent fragments of the $\sim 860-1,725 \mathrm{nt}$ region of the $18 \mathrm{~S} \mathrm{rRNA}$, the present material is most closely related to an unnamed species (GenBank: KC800702) isolated from P. semivariegatus from Swaziland (96.3-96.6\% identity, $\mathrm{p}=0.03-0.04)$, to an unnamed species (GenBank:
KC800703) from Python natalensis Smith, from Swaziland (96.3-96.6\% identity, $\mathrm{p}=0.03$ ) and an unnamed species (GenBank: KC866368) from Dendroaspis polylepis Günther in Swaziland or Tanzania $(96.3-96.6 \%$ identity, $\mathrm{p}=0.03-0.04)$ (Haklová et al. 2014).

\section{Description of peripheral blood stages of Hepatozoon species}

Hepatozoon angeladaviesae $\mathrm{sp} . \mathrm{n}$.

Fig. $1 \mathrm{~A}-\mathrm{C}$

ZooBank number for species:

urn:Isid:zoobank.org:act:C04EDDB3-8E91-4AB7-8361-4B1FA25D87CF

Mature gamonts $(\mathrm{n}=50)$ : Lying singly within mature erythrocytes, parasitophorous vacuole (PV) rarely evident, but appearing as thin 'halo' when visible (Fig. 1A), 14.1-17.3 (16.0 \pm 0.7$)$ long, 4.8-6.5 (5.8 \pm 0.5$)$ wide, straighter than curved, one pole faintly pointed (arrowhead) (Fig. 1A,C), opposite pole rounded, sometimes appearing folded (arrow) (Fig. 1C), cytoplasm staining blue. 


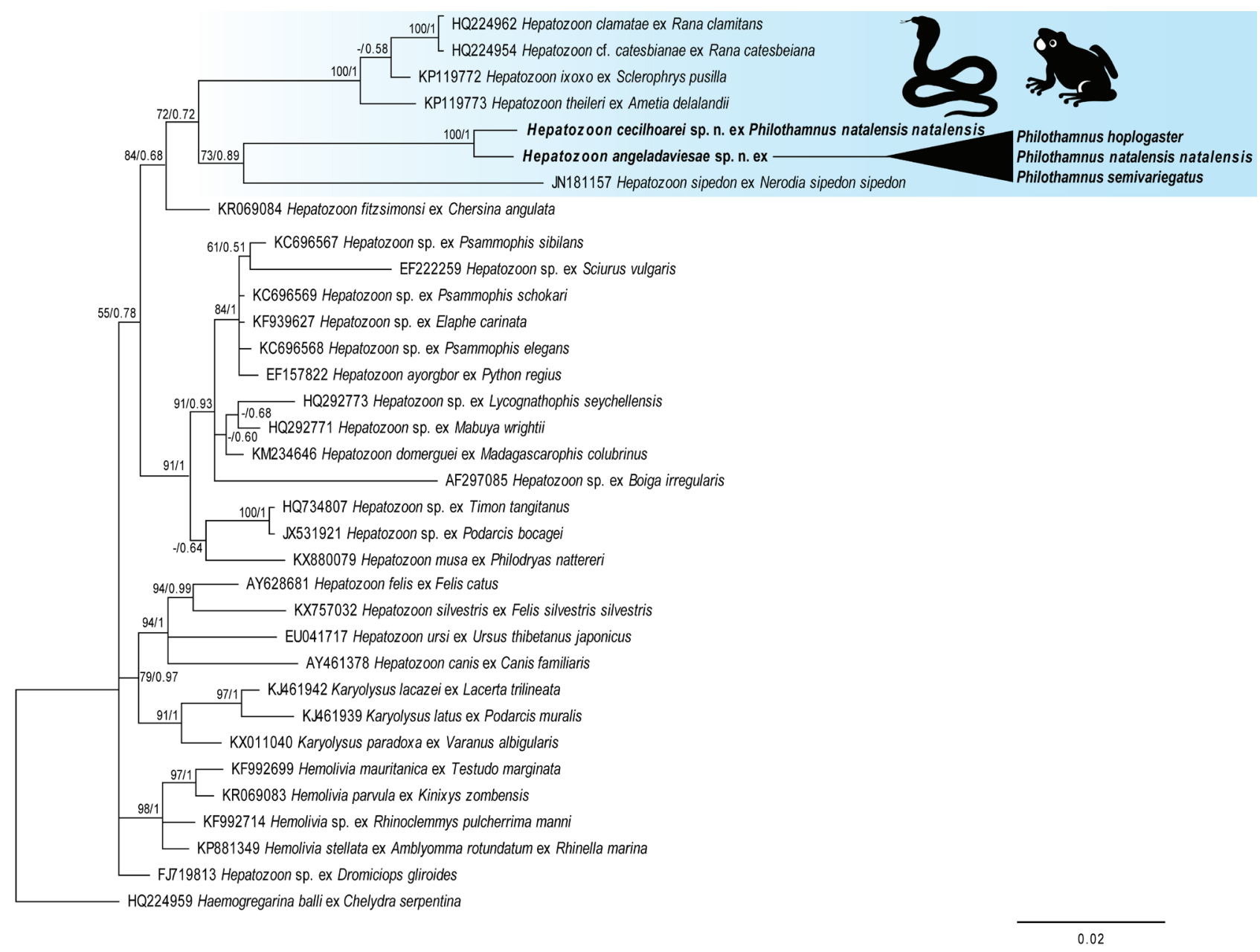

Fig. 2. Maximum Likelihood (ML) and Bayesian Inference (BI) analysis of Hepatozoon angeladaviesae sp. n. and Hepatozoon cecilhoarei sp. n. from Philothamnus spp. and their relationships with other haemogragarins, based on partial 18S rDNA sequences. Tree topologies for both the ML and BI trees were identical; the nodal support values, bootstrap for ML and posterior probability for BI, are represented as $\mathrm{ML} / \mathrm{BI}$ on the $\mathrm{ML}$ tree.

Table 2. Evolutionary differences of species of Hepatozoon Miller, 1908 isolated from snakes of Africa not included in the phylogenetic analysis presented in Fig. 2, representing the $\sim 860-1,725 \mathrm{nt}$ region and expressed as percent similarity (bottom left) and pair-wise distance ( $\mathrm{p}$-distance) (top right).

\begin{tabular}{|c|c|c|c|c|c|c|c|c|c|c|c|c|}
\hline & $\begin{array}{l}\text { Accession } \\
\text { number }\end{array}$ & Hepatozoon species & Host species & 1 & 2 & 3 & 4 & 5 & 6 & 7 & 8 & 9 \\
\hline 1 & $\begin{array}{l}\text { MG519501- } \\
\text { MG519503 }\end{array}$ & $\begin{array}{l}\text { Hepatozoon angeladaviesae } \\
\text { sp. n. }\end{array}$ & $\begin{array}{l}\text { Philothamnus hoplogaster (Günther), } \\
\text { Philothamnus natalensis natalensis (Smith), } \\
\text { Philothamnus semivariegatus (Smith) }\end{array}$ & & 0.01 & 0.04 & 0.03 & 0.05 & 0.04 & 0.03 & 0.03 & 0.04 \\
\hline 2 & MG519504 & Hepatozoon cecilhoarei sp. n. & Philothamnus natalensis natalensis & 99.5 & & 0.03 & 0.03 & 0.04 & 0.03 & 0.03 & 0.03 & 0.04 \\
\hline 3 & KC800702 & Hepatozoon sp. & Philothamnus semivariegatus & 96.3 & 96.6 & & 0.01 & 0.02 & 0.01 & 0.01 & 0.01 & 0.01 \\
\hline 4 & KC800703 & Hepatozoon sp. & Python natalensis Smith & 96.3 & 96.6 & 99.2 & & 0.02 & 0.01 & 0.00 & 0.00 & 0.00 \\
\hline 5 & KC866369 & Hepatozoon sp. & Dendroaspis jamesoni kaimosae Loveridge & 95.1 & 95.4 & 97.7 & 97.4 & & 0.02 & 0.02 & 0.02 & 0.03 \\
\hline 6 & KC866368 & Hepatozoon sp. & Dendroaspis polylepis Günther & 96.3 & 96.6 & 99.5 & 99.2 & 98.2 & & 0.01 & 0.01 & 0.01 \\
\hline 7 & KC866370 & Hepatozoon sp. & Dendroaspis jamesoni jamesoni (Traill) & 96.0 & 96.3 & 98.8 & 99.3 & 97.4 & 98.8 & & 0.00 & 0.00 \\
\hline 8 & KC800704 & Hepatozoon sp. & Gonionotophis capensis capensis (Smith) & 96.2 & 96.5 & 99.0 & 99.9 & 97.3 & 99.0 & 99.2 & & 0.00 \\
\hline 9 & JQ746622 & $\begin{array}{l}\text { Hepatozoon garnhami } \\
\text { (Mohammad, Ramdan, } \\
\text { Mohammed et Fawzi, 1996) }\end{array}$ & Psammophis schokari (Forskal) & 96.0 & 96.3 & 98.9 & 99.5 & 97.1 & 98.9 & 99.0 & 99.3 & \\
\hline
\end{tabular}

Nucleus 3.6-5.7 (5.0 \pm 0.5$)$ long, 3.7-5.8 (4.7 \pm 0.5$)$ wide, staining dark purple-pink with compactly arranged chromatin, situated almost centrally with mid-nucleus to anterior measuring 6.4-8.5 (7.4 \pm 0.5$)$, mid-nucleus to posterior 6.5-9.4 (8.5 \pm 0.6$)$, square (Fig. 1B) to rounded (Fig. 1C).
Effects on host cell: Normal erythrocytes $(\mathrm{n}=50)$ measure 14.3-19.4 $(16.0 \pm 0.9) \times 9.2-13.4(11.7 \pm 0.7)$, compared to significantly elongated $(\mathrm{P}<0.01)$ parasitised erythrocytes $(\mathrm{n}=50)$ measure 19.6-31.3 $(25.4 \pm 2.2) \times$ $9.4-16.1(12.2 \pm 1.5)$. Normal erythrocyte nucleus meas- 
ures 5.5-8.1 $(6.7 \pm 0.6) \times 2.7-4.5(3.7 \pm 0.4)$, compared to significantly elongated $(\mathrm{P}<0.01)$ and condensed $(\mathrm{P}<0.01)$ parasitised host cell nucleus measuring $5.7-13(10.3 \pm 1.2)$ $\times 1.7-3.6(2.7 \pm 0.4)$. Host cell nucleus markedly displaced and condensed, sometimes central and parallel to gamont (Fig. 1B) or to one pole of gamont showing slight degree of karyolysis, but no evidence of vacuolation, in some cases karyorrhexis (Fig. 1C). Dehaemoglobinisation and hypertrophy of host cell evident, resulting in a 'wafer-thin' undulating cell membrane.

Type host: Philothamnus semivariegatus (Smith) (Ophidia: Colubridae).

Other hosts: Philothamnus hoplogaster (Günther), Philothamnus natalensis natalensis (Smith) (Ophidia: Colubridae).

Vector: Unknown.

Type locality: Ndumo Game Reserve (26 52'46"S; $\left.32^{\circ} 15^{\prime} 25^{\prime \prime E}\right)$, KwaZulu-Natal, South Africa.

Type specimens: Hapantotype, deposited in the Protozoan collection of the National Museum, Bloemfontein, South Africa, voucher specimen number: NMB P 440; sequences uploaded onto GenBank, $18 \mathrm{~S}$ sequence accession numbers: MG519501-MG519503.

Etymology: The species is named after Angela Josephine Davies (1947-2013), to commemorate her contribution to the knowledge of parasitic protozoa in vertebrates, as well as her singular dedication to and enthusiasm in sharing this knowledge with all those she mentored.

Remarks. During the early to mid 1900s, haemogregarines were reported parasitising Philothamnus spp. from Equatorial to Saharan Africa. However, most of these reports based solely on peripheral gamont stages that lacked descriptive detail. Furthermore, none of these reports led to a complete description in which the haemogregarines were named. Accounts included a report from a $P$. semivariegatus as well as an unidentified Philothamnus sp. in French West Africa, from an unidentified Philothamnus sp. in Mabira, Uganda, from a Philothamnus irregularis Leach in Stanleyville, Democratic Republic of the Congo and Gambia and lastly from a $P$. irregularis in Nairobi, Kenya (Bouet 1909, Hoare 1920, Schwetz 1931, Garnham and Duke 1953, Ball 1967). Those providing enough detail for a more thorough comparison included the reports of Hoare (1920) and Ball (1967).

Hoare (1920) described a parasite that caused the dehaemoglobinisation of the host cell, the length of which is very similar to that of $H$. angeladaviesae sp. n. described in the present study, but the width was described as considerably narrower (mean width $2.3 \mu \mathrm{m}$ ) compared to the present material (mean width $5.4 \mu \mathrm{m}$ ). The slender form of the parasite and the slight karyolysis of the parasite on the host cell nucleus described by Hoare (1920) led him to suggest its assignment as a species of Karyolysus.

The haemogregarine gamont stages described by Ball (1967) were found to be narrower (mean width $3.8 \mu \mathrm{m}$ ) than the gamonts of $H$. angeladaviesae and, in contrast, were not described as causing any dehaemoglobinisation of the host cell. The morphological dissimilarities between the gamont stages described by Hoare (1920), Ball (1967) and those of $H$. angeladaviesae draw us to the conclusion that they do not belong to the same species.

In comparison to described peripheral gamont stages of species of Hepatozoon described from other South African snakes, such as those $H$. bitis and $H$. refringens parasitising Bitis arietans (Viperidae) and Pseudoaspis cana (Lamprophiidae), respectively, the gamonts of $H$. angeladaviesae are much larger, both in length and width (Table 1) with the characteristic dehaemoglobinisation of the host cell, which is not evident in the other two Hepatozoon spp. Furthermore, $H$. angeladaviesae does not conform in size, morphology and effects on host cells of other formally described Hepatozoon species of snakes from Africa (Table 1).

Hepatozoon cecilhoarei $\mathrm{sp} . \mathrm{n}$.

Fig. 1D-F

ZooBank number for species:

urn:1sid:zoobank.org:act:91129F0E-3247-4169-B0F2-C8AED06AF255

Mature gamonts $(\mathrm{n}=18)$ : Occurr singly, within larger PV (Fig. 1D), within mature erythrocytes, 13.1-15.9 (14.9 \pm 0.7$)$ long, $2.1-2.7(2.3 \pm 0.2)$ wide, gamont slightly curved within curved PV. PV forming hook-like point at one pole (arrowhead) (Fig. 1E,F), other pole rounded, PV showing irregular pink granular deposits; gamont cytoplasm staining blue; nucleus with tightly arranged chromatin, essentially central with mid-nucleus to anterior 5.1-8.0 $(6.8 \pm 0.7)$, mid-nucleus to posterior 7.4-10.5 (8.9 \pm 0.8$)$, rectangular, measuring $4.2-5.8(5.1 \pm 0.5)$ long, $1.2-1.6$ $(1.3 \pm 0.1)$ wide, staining dark purple.

Effects on host cell: Normal erythrocytes $(n=18)$ measure 14.4-19.4 $(16.1 \pm 1.2) \times 9.2-12.8(11.4 \pm 0.8)$, compared to significantly elongated and narrowed $(\mathrm{P}<0.01)$ parasitised host cells $(\mathrm{n}=18)$ measuring 16.2-19.3 $(17.4 \pm 0.8) \times 7.6-10.9(8.8 \pm 0.9)$. Normal erythrocyte nucleus measures 5.7-7.9 $(6.9 \pm 0.5) \times 3.0-4.3(3.8 \pm 0.3)$, compared to significantly elongated and condensed $(\mathrm{P}<0.01)$ parasitised host cell nucleus measuring $8.8-10.4$ $(9.6 \pm 0.5) \times 2.7-3.9(3.1 \pm 0.3)$. Host cell nucleus displaced to central and parallel region of gamont, host nucleus condensed showing slight degree of karyolysis, but with no vacuoles observed; evident dehaemoglobinisation of host cell cytoplasm.

Type host: Philothamnus natalensis natalensis (Smith) (Ophidia: Colubridae).

Other hosts: Philothamnus hoplogaster (Günther) (Ophidia: Colubridae).

Vect or: Unknown.

Type 1ocality: Ndumo Game Reserve (265' $46^{\prime \prime}$; $\left.32^{\circ} 15^{\prime} 25^{\prime \prime E}\right)$, KwaZulu-Natal, South Africa.

Other 1 ocality: Mabira $\left(00^{\circ} 23^{\prime} 54^{\prime \prime N}\right.$; $\left.33^{\circ} 00^{\prime} 59^{\prime \prime E}\right)$, Uganda.

Type specimens: Hapantotype, deposited in the Protozoan collection of the National Museum, Bloemfontein, South Africa, voucher specimen number: NMB P 441 sequences uploaded onto GenBank, 18S sequence accession number: MG519504. 
Etymology: The species is named after Cecil Arthur Hoare (1892-1984), an eminent parasitologist who did extensive research on the protozoan parasites of African herpetofauna, and whom it is believed by the authors to have first discovered, but not named, this parasite.

Remarks. In comparison to the gamont stages of $H$. angeladaviesae sp. n. (average $16.0 \mu \mathrm{m} \times 5.8 \mu \mathrm{m}$ ), those of H. cecilhoarei sp. $\mathrm{n}$. are shorter (mean length $14.9 \mu \mathrm{m}$ ) and narrower (mean width $2.3 \mu \mathrm{m}$ ). Furthermore, gamonts of $H$. cecilhoarei do not cause the same enlargement that results in an 'undulating' effect on the host cell, nor do they cause the same karyolysis that leads to the fragmentation of the host cell nucleus. Similarly, as with the gamonts of $H$. angeladaviesae, $H$. cecilhoarei does not conform in size, morphology or effect on the host cell to other species of Hepatozoon formally described and named, which parasitise other snake species in Africa.

Hepatozoon cecilhoarei, however, does closely resemble to two haemogregarines described, but not named, from other Philothamnus spp. in Africa. As mentioned previously, Hoare (1920) reported a haemogregarine from an unidentified species of Philothamnus from Uganda. This parasite was described to be $15.0-16.0 \mu \mathrm{m} \times 2.3 \mu \mathrm{m}$ in size, closely conforming to the length and width of H. cecilhoarei. Hoare (1920) also described the gamonts of the haemogregarine he discovered as elongate and slender, with the ends or poles of the gamont slightly bent inwards, but never bent over on the gamont itself. In addition, he described the parasite gamont to usually attain the same length as that of the host cell, or occasionally they could be longer than that of the host cell. As such, in form, the gamont of the haemogregarine described by Hoare (1920) compares closely to that of $H$. cecilhoarei described in the present study.

Considering the effects on the host cell when comparing the description of Hoare (1920) to that of the present study, both haemogregarine gamonts cause host cell alteration, decreasing host cell size and causing dehaemoglobinisation, as well as the host nucleus to become hypertrophied and elongated. In both the present study and that of Hoare (1920), mature gamonts were the only stages identified. Furthermore, the gamont lay parallel to the long axis of the host cell adjacent to the host cell nucleus showing some degree of karyolysis.

However, besides Hoare's (1920) mentioning the slight karyolysis of the host cell nucleus, which caused the chromatin to appear entangled in irregular accumulations and strands, he also mentioned that vacuoles were sometimes present. This was not evident in the present study, but it may be a result of a less mature or developed infection. Hoare (1920) also never observed an evident parasitophorous vacuole or irregular pink granules as was notable in a number of gamonts of $H$. cecilhoarei. Regardless, the overall similarity of the haemogregarine described by Hoare (1920) and that of the present study, suggest strongly that they are the same.

\section{DISCUSSION}

The genus Philothamnus falls within the family Colubridae, including largely arboreal snakes, which often occupy habitats near to water (Bates et al. 2014). As mentioned previously, the only species of Hepatozoon described from South African snakes to date include two from the families Viperidae and Lamprophiidae, respectively, the two species of Hepatozoon described in this paper thus representing the first taxa of this genus reported from the family Colubridae in South Africa (Sambon and Seligmann 1907, Fantham 1925, Van As et al. 2013). Since the ecology of snakes has formerly been demonstrated as important with regards to their associated diversity of Hepatozoon spp. (Smith et al. 1994, Telford et al. 2001, Telford 2009), it is not surprising that the species infecting Philothamnus spp. in this study are different (based on morphology) from those occuring in snakes of the genera of Bitis Gray and Pseudaspis Fitzinger.

Past studies have shown habitat and the resulting diet of snakes important, particularly where more than one species of intermediate host is involved (Smith et al. 1994, Telford et al. 2001, Telford 2009). Hepatozoon sipedon, for example, is known to naturally infect the snake, Nerodia sipedon sipedon, through the ingestion of an anuran, Rana pipiens (Schreber) (Smith 1994, Netherlands et al. 2014a). Furthermore, these non-ophidian intermediate host associations are becoming evident in the phylogenetic analyses of species of Hepatozoon because species infecting snakes with a diet comprising mostly anurans and those with a diet comprising mostly saurians belong to separate lineages (Haklová et al. 2014).

Tomé et al. (2013) demonstrated that diet appears to be a key element for infection of snakes by species of Hepatozoon. The authors found that the lineages infecting saurophagous snakes of the genus Psammophis Fitzinger clustered together with those from different types of lizards that form a large portion of the diet of these snakes. Both $B$. arietans and $P$. cana fulfill ecologically different roles, being more terrestrial and feeding mostly on rodents, compared to species of Philothamnus, which are arboreal, preferring to occupy a habitat nearer to water, feeding on lizards, frogs, fish and nestling birds (Bates et al. 2014). Since all six specimens of Philothamnus examined in the present study were found near temporary and permanent pans with a large population of frogs that provide a readily available food source and potential source of infection, and both $H$. angeladaviesae sp. n. and $H$. cecilhoarei $\mathrm{sp} . \mathrm{n}$. were found to fall into a clade comprising snake and anuran species of Hepatozoon (Fig. 2), it would be beneficial to identify possible frog hosts, but also locate fresh specimens of $H$. bitis and $H$. refringens so that they too may be compared in phylogenetic analyses.

It is unfortunate that the species of Hepatozoon parasitising P. semivariegatus from Swaziland sequenced by Haklová et al. (2014) could not be compared on a morphological basis to $H$. angeladaviesae and $H$. cecilhoarei. Furthermore, as the fragment used in the evolutionary 
divergence estimates represents a relatively conservative section of the 18S rRNA gene, results may demonstrate a closer relatedness than what is truly the case. However, it does emphasise the need to provide descriptions based on a combination of morphology and molecular data, which has been highlighted by numerous authors, e.g. Cook et al. (2016), Tomé et al. (2016) and Borges-Nojosa et al. (2017). Moreover, it is anticipated that a multigene approach will prove useful in resolving the diversity, phylogeny and taxonomy of the paraphyletic Hepatozoon, as has been used in the analyses of the haemoproteids and eimeriid coccidia (Pineda-Catalan et al. 2013, Ogedengbe et al. 2015).

Acknowledgements. We would like to thank Ezemvelo KwaZulu-Natal Wildlife for permission to sample reptiles within Ndumo
Game Reserve. In addition, we would like to thank Microbiology, Unit for Environmental Sciences and Management, North-West University (NWU), Potchefstroom campus, for use of their facilities. The financial assistance of the South African National Research Foundation (NRF) towards CAC is also hereby acknowledged (project SFP13090332476), and in part to ECN who was supported by the DAAD-NRF doctoral scholarship (Grant UID: 108803), and the VLIR-UOS university scholarship (ID 0620854/Contract 000000076310). JVA was supported by a NRF Thutuka grant (TTK 14042266483) and NJS by a NRF Incentive Funding for Rated Researchers grant (IFR 170210222411). Opinions expressed and conclusions arrived at are those of the authors and are not necessarily to be attributed to the NRF. This is contribution No. 241 from the NWU-Water Research Group. We are most grateful to Maarten P.M. Vanhove from the Biology Department, Royal Museum for Central Africa (Belgium), for obtaining the work of Schweitz (1931).

\section{REFERENCES}

AbDel-Baki A.S., Al-Quraishy S., Zhang J.Y. 2014: Redescription of Haemogregarina garnhami (Apicomplexa: Adeleorina) from the blood of Psammophis schokari (Serpentes: Colubridae) as Hepatozoon garnhami n. comb. based on molecular, morphometric and morphologic characters. Acta Parasitol. 59: 294-300.

BALL G.H. 1967: Blood sporozoans from East African Amphibia. J. Eukaryot. Microbiol. 14: 521-527.

Barta J.R., Ogedengbe J.D., Martin D.S., Smith T.G. 2012 Phylogenetic position of the adeleorinid coccidia (Myzozoa, Apicomplexa, Coccidia, Eucoccidiorida, Adeleorina) inferred using 18S rDNA sequences. J. Eukaryot. Microbiol. 59: 171-180.

Bashtar A.R., AbDel-Ghaffar F.A. 1987: Light microscope study on the life cycle of Haemogregarina najae infecting the snake Naja nigricollis nigricollis (Elapidae, Proteroglypha, Squamata) from Egypt. Proc. Zool. Soc. A. R. Egypt 14: 33-44.

Bashtar A.R., Abdel-Ghaffar F.A., Shazly M.A. 1991: Life cycle of Hepatozoon mehlhorni sp. nov. in the viper Echis carinatus and the mosquito Culex pipiens. Parasitol. Res. 77: 402-410.

Bashtar A.R., Boulos R., Mehlhorn H. 1984: Hepatozoon aegypti nov. sp. Parasitol. Res. 70: 29-41.

Bates M.F., Branch W.R., Bauer A.M., Burger M., Marais J., Alexander G.J., De Villiers M.S. 2014: Atlas and Red List of the Reptiles of South Africa, Lesotho and Swaziland. Suricata 1. South African National Biodiversity Institute, Pretoria, pp. 485.

Billet M.A. 1904: Sur une hémogrégarine karyolysante de la couleuvre vipérine. C. R. Hebd. Séances Mém. Soc. Biol. 1: 484-485.

Borges-Nojosa D.M., Borges-Leite M.J., Maia J.P., Zanchi-Silva D., Da Rocha Braga R., Harris D.J. 2017: A new species of Hepatozoon Miller, 1908 (Apicomplexa: Adelerina) from the snake Philodryas nattereri Steindachner (Squamata: Dipsadidae) in northeastern Brazil. Syst. Parasitol. 94: 65-72.

Bouet J. 1909: Hemogrégarines de L’Afrique occidentale français. C. R. Séances Soc. biol. 66: 43-45.

Cook C.A., Lawton S.P., Davies A.J., Smit N.J. 2014a: Reassignment of the land tortoise haemogregarine Haemogregarina fitzsimonsi Dias 1953, (Adeleorina: Haemogregarinidae) to the genus Hepatozoon Miller 1908 (Adeleorina: Hepatozoidae) based on parasite morphology, life cycle and phylogenetic analysis of $18 \mathrm{~S}$ rDNA sequence fragments. Parasitology 141: 1611-1620.

Cook C.A., Netherlands E.C., Smit N.J. 2016: Redescription, molecular characterization and taxonomic re-evaluation of a unique African monitor lizard haemogregarine Karyolysus paradoxa (Dias, 1954) n. comb. (Karyolysidae). Parasit. Vectors 9: 347.

Cook C.A., Smit N.J., Davies A.J. 2009: A redescription of Haemogregarina fitzsimonsi Dias, 1953 and some comments on Haemogregarina parvula Dias, 1953 (Adeleorina: Haemogregarinidae) from southern African tortoises (Cryptodira: Testudinidae), with new host data and distribution records. Folia Parasitol. 56: 173-179.

Cook C.A., Smit N.J., Davies A.J. 2014b: First record of an intraleucocytic haemogregarine (Adeleorina: Haemogregarinidae) from South African tortoises of the species Stigmochelys pardalis (Cryptodira: Testudinidae). Afr. Zool. 49: 290-294.

Criado-Fornelio A., Ruas J.L., Casado N., Farias N.A.R., Soares M.P., Müller G., Brum J.G.W., Berne M.E.A., Buling-Saraña A., BArba-CArretero J.C. 2006: New molecular data on mammalian Hepatozoon species (Apicomplexa: Adeleorina) from Brazil and Spain. J. Parasitol. 92: 93-99.

Darriba D., Taboada G.L., Doallo R., Posada D. 2012: jModelTest 2: more models, new heuristics and parallel computing. Nat. Methods. 9: 772 .

DiAS J.A.T.S. 1952: Resultados de um reconhecimento zoológico no alto Limpopo efectuado pelos Drs. F. Zumpt. Moç. Doc. Trimest. 71: 61-79.

Dias J.A.T.S. 1953: Subsídios para o estudo dos hematozoários dos répteis de Moçambique. Bol. Soc. Est. Moç. 82: 3-19.

EDGAR R.C. 2004: MUSCLE: multiple sequence alignment with high accuracy and high throughput. Nucl. Acids Res. 5: 17921797.

Fantham H.B. 1925: Some parasitic protozoa found in South Africa. - VIII. S. Afr. J. Sci. 22: 346-354.

Garnham P.C.C. 1950: Blood parasites of East African vertebrates, with a brief description of exo-erythrocytic schizogony in Plasmodium pitmani. Parasitology 40: 328-337.

Garnham P.C.C., Duke B.O.L. 1953: Certain parasitic Protozoa from the Gambia. Trans. Roy. Soc. Trop. Med. H. 47: 7-8.

Guindon S., Gascuel O. 2003: A simple, fast and accurate method to estimate large phylogenies by maximum-likelihood. Syst. Biol. 52: 696-704.

Haklová B., Majláthová V., Majláth I., Harris D.J., Petrilla V., Litschka-Koen T., Oros M., Peт̌ko B. 2014: Phylogenetic relationship of Hepatozoon blood parasites found in snakes from Africa, America and Asia. Parasitology 141: 389-398.

Haklová-Kočíková B., Hižňanová A., Majláth I., RačKa K., Harris D.J., Földvári G., Tryjanowski P., Kokošová 
N., Malčeková B., Majláthová V. 2014: Morphological and molecular characterization of Karyolysus - a neglected but common parasite infecting some European lizards. Parasit. Vectors 7: 555.

HoAre C.A. 1920: On some new haemogregarines from British East Africa. Parasitology 12: 315-327.

HoAre C.A. 1932: On protozoal blood parasites collected in Uganda. Parasitology 24: 210-224.

HuElSENBECK J.P., RonQuist F. 2001: MRBAYES: Bayesian inference of phylogenetic trees. Bioinformatics 17: 754-755.

Karadjian G., Chavatte J., Landau I. 2015: Systematic revision of the adeleid haemogregarines, with creation of Bartazoon n. g., reassignment of Hepatozoon argantis, 1954 to Hemolivia, and molecular data on Hemolivia stellata. Parasite 22: 31.

Kearse M., Moir R., Wilson A., Stones-Havas S., Cheung M., Sturrock S., Buxton S., Cooper A., Markowitz S., Duran C., Thierer T., Ashton B., Mentuies P., DrumMOND A. 2012: Geneious Basic: an integrated and extendable desktop software platform for the organization and analysis of sequence data. Bioinformatics 28: 1647-1649.

Kumar S., Stecher G., Tamura K. 2016: MEGA7: Molecular Evolutionary Genetics Analysis Version 7.0 for Bigger Datasets. Mol. Biol. Evol. 33: 1870-1874.

KVičerová J., Hypša V., DVoŘáková N., Mikulíčé P., Jandzik D., George Gardner M., Javanbakht H., Tiar G., ŠIroKÝ P. 2014: Hemolivia and Hepatozoon: haemogregarines with tangled evolutionary relationships. Protist 165: 688-700.

LAVERAN A. 1902: Sur quelques haemogregarines des ophidiens. C. R. Hebd. Séances Acad. Sci. 135: 1036-1040.

LaVeran A. 1905: Contribution a l'étude des grandes haemogregarines des grenouilles. C. R. Soc. Séances Soc. Biol. 59: 172 175 .

Laveran A., Pettit A. 1909: Sur une hémogrégarine du Python sebai. C. R. Hebd. Séances Acad. Sci. 148: 1142-1146.

Laveran A., Pettit A. 1911: Sur une hémogrégarine de la vipère à cornes. C. R. Hebd. Séances Acad. Sci. Biol. 63: 95-96.

Maia J.P., Carranza S., Harris D.J. 2016: Comments on the systematic revision of adeleid haemogregarines: is more data needed? J. Parasitol. 102: 549-552.

Manceaux L. 1908: Hemogregarines de Zamensis hippocrepis et de Zamensis algirus. Arch. Inst. Pasteur, Tunis 4: 186-189.

Mathew J.S., Van Den Bussche R.A., Ewing S.A., Malayer J.R., Latha B.R., Panciera R.J. 2000: Phylogenetic relationships of Hepatozoon (Apicomplexa: Adeleorina) based on molecular, morphologic, and life-cycle characters. J. Parasitol. 86: $366-372$

Medlin L., Elwood H.J., Stickel S., Sogin M.L. 1988: The characterization of enzymatically amplified eukaryotic 16S-like rRNA-coding regions. Gene 71: 491-499.

Mohammad F.A.S., Ramdan N.F., Mohammed S.H., Fawz S.M. 1996: On two haemogregarines (Protozoa: Apicomplexa) from colubrid and Elapidae snakes in Egypt. Qatar Univ. Sci. J. 16: $127-139$.

Morsy K., Bashtar A.R., Abdel-Ghaffar F., Al-Quraishy S., Al-Hashimi S., Al-Ghamdi A., Shazly M. 2013: Development stages of Hepatozoon seurati (Laveran and Pettit 1911) comb. nov., a parasite of the corned viper Cerastes cerastes and the mosquito Culex pipiens from Egypt. Parasitol. Res. 112: $2533-2542$

Netherlands E.C., Cook C.A., Du Preez L.H., Vanhove M.P.M, Brendonck L., Smit N.J. 2018: Monophyly of the species of Hepatozoon (Adeleorina: Hepatozoidae) parasitizing (African) anurans, with the description of three new species from hyperoliid frogs in South Africa. Parasitology: (in press).

Netherlands E.C., Cook C.A., Kruger D.J.D, Du Preez L.H., Sмit N.J. 2015: Biodiversity of frog haemoparasites from sub-tropical northern KwaZulu-Natal, South Africa. Int. J. Parasitol. Parasites. Wildl. 4: 135-141.
Netherlands E.C., Cook C.A., Smit N.J. 2014b: Hepatozoon species (Adeleorina: Hepatozoidae) of African bufonids, with morphological description and molecular diagnosis of Hepatozoon ixoxo sp. nov. parasitising three Amietophrynus species (Anura: Bufonidae). Parasit. Vectors 7: 552.

Netherlands E.C., Cook C.A., Smit N.J., Du Preez L.H. 2014a: Redescription and molecular diagnosis of Hepatozoon theileri (Laveran, 1905) (Apicomplexa: Adeleorina: Hepatozoidae), infecting Amietia quecketti (Anura: Pyxicephalidae). Folia Parasitol. 61: 293-300.

Ogedengbe J.D., Ogedengbe M.E., Hafeez M.A., Barta J.R. 2015: Molecular phylogenetics of eimeriid coccidia (Eimeriidae, Eimeriorina, Apicomplexa, Alveolata): a preliminary multi-gene and multi-genome approach. Parasitol. Res. 114: 4149-4160.

Peirce M.A. 1984: Some parasites of reptiles from Zambia and Indian Ocean islands with a description of Haemogregarina zambiensis sp. n. from Dispholidus typus (Colubridae). J. Nat. Hist. 18: 211-217.

Phisalix M. 1914: Sur une hémogregarine nouvelle, parasite de Boodon fuliginosus Boie, et ses formes de multiplication endogene. Bull. Soc. Pathol. Exot. 7: 575-577.

Pineda-Catalan O., Perkins S.L., Peirce M.A., Engstrand R., Garcia-Davila C., Pinedo-Vasquez M., Alonso AguIRRE A. 2013: Revision of hemoproteid genera and description and redescription of two species of chelonian hemoproteid parasites. J. Parasitol. 99: 1089-1098.

Sambon L.W., Seligmann C.G. 1907: Descriptions of five new species of haemogregarines from snakes. Proc. General Meetings for Scientific Business of the Zoological Society of London, London, March 1907. Messrs. Longmans, Green, and Co., London, pp. 283-284.

Sambon L.W., Seligmann C.G. 1909: The haemogregarines of snakes. T. Pathol. Soc. Lond. 58: 310-370.

Schweitz J. 1931: Les hématozoaires des serpents de Stanleyville (Congo Belge). Ann. Parasitol. 9: 303-310.

Sloboda M., Kamler M., Bulantová J., VotÝpka J., Modrí D. 2007: A new species of Hepatozoon (Apicomplexa: Adeleorina) from Python regius (Serpentes: Boidae) and its experimental transmission by a mosquito vector. J. Parasitol. 93: 1189-1198.

Sмiтн T.G. 1996: The genus Hepatozoon (Apicomplexa: Adeleina). J. Parasitol. 82: 565-585.

Smith T.G., Desser S.S., Martin D.S. 1994: The development of Hepatozoon sipedon sp. nov. (Apicomplexa: Adeleina: Hepatozoidae) in its natural host, the northern water snake (Nerodia sipedon sipedon), in the culicine vectors Culex pipiens and $C$. territans, and in an intermediate host, the northern leopard frog (Rana pipiens). Parasitol. Res. 80: 559-568.

Telford S.R. 2009: Hemoparasites of the Reptilia: Color Atlas and Text. CRC Press, New York, 376 pp.

Telford S.R., Wozniak E.J., Butler J.F. 2001: Haemogregarine specificity in two communities of Florida snakes, with descriptions of six new species of Hepatozoon (Apicomplexa: Hepatozoidae) and a possible species of Haemogregarina (Apicomplexa: Haemogregarinidae). J. Parasitol. 87: 890-905.

Tomé B., Maia J.P.M.C, HaR RIS D.J. 2013. Molecular assessment of apicomplexan parasites in the snake Psammophis from North Africa: do multiple parasite lineages reflect the final vertebrate host diet? J. Parasitol. 99: 883-887.

Tomé B., Rato C., Harris D.J., Perera A. 2016: High diversity of Hepatozoon spp. in geckos of the genus Tarentola. J. Parasitol. 102: 476-480.

Ujvari B., Madsen T., Olsson M. 2004: High prevalence of Hepatozoon spp. (Apicomplexa: Hepatozoidae) infection in water pythons (Liasis fuscus) from tropical Australia. J. Parasitol. 90: 670-672.

Van As J., Davies A.J., Smit N.J. 2013: Hepatozoon langii n. sp. and Hepatozoon vacuolatus n. sp. (Apicomplexa: Adeleorina: Hepatozoidae) from the crag lizard (Sauria: Cordylidae) Pseu- 
docordylus langi from the North Eastern Drakensberg escarpment, Eastern Free State, South Africa. Zootaxa 3608: 345-356.

Van As J., Davies A.J., Smit N.J. 2015: Life cycle of Hepatozoon

affluomaloti sp. n. (Apicomplexa: Haemogregarinidae) in crag lizards (Sauria: Cordylidae) and in culicine mosquitoes from South Africa. Folia Parasitol. 62: 008.
Received 23 June 2017

Accepted 10 January 2018
Published online 3 April 2018

Cite this article as: Cook C.A., Netherlands E.C., van As J., Smit N.J. 2018: Two new species of Hepatozoon (Apicomplexa: Hepatozoidae) parasitising species of Philothamnus (Ophidia: Colubridae) from South Africa. Folia Parasitol. 65: 004. 